\title{
First-principles investigation of Co wires at Pt(111) step-edges
}

\author{
S. Baud ${ }^{a}$ G. Bihlmayer ${ }^{b, *}$ S. Blügel ${ }^{b}$ Ch. Ramseyer ${ }^{a}$ \\ ${ }^{a}$ Laboratoire de physique moléculaire, UMR CNRS 6624, Université de Franche-Comté, F-25030 Besançon Cedex, France \\ b Institut für Festkörperforschung, Forschungszentrum Jülich, D-52425 Jülich, Germany
}

\begin{abstract}
We report on ab initio calculations of $\mathrm{Pt}(111)$ B-type step-edges decorated with Co chains of different thickness. As found experimentally, these Co $n$-wires show for $n=1$ (monowire) a large magnetic anisotropy that decreases for larger $n$ in a non-monotonous way. Also the easy axis shows a oscillatory behavior with increasing $n$ that can be traced back to competing effects arising from different strands of the thicker wires.
\end{abstract}

Key words: ab initio calculations, nanowires, magnetic anisotropy

PACS: 73.20.-r, 75.30.Gw, 75.75.+a

\section{Introduction}

Transition-metal (TM) decorated step edges, e.g. of vicinal surfaces, are a fascinating natural laboratory for the physics of low-dimensional, sometimes magnetic, systems. Quasi one-dimensional magnets have shown many unique properties, like very large orbital moments and large magnetic anisotropy energies (MAE) as a consequence. For the magnetic order in the one-dimensional system, the anisotropy is a determining factor. It is the magnetocrystalline anisotropy energy that allows us to study the magnetic properties of these systems at finite temperatures.

Due to their low symmetry, decorated step edges also allow the possibility to have easy magnetic axes in many different directions. If the easy axis

\footnotetext{
* Corresponding author

Email address: G.Bihlmayer@fz-juelich.de

(G. Bihlmayer).
}

of magnetization is not oriented along the stepedge, in principle every direction perpendicular to this direction can be a favored magnetization axis. For a single Co chain on the B-type step-edge of a $\operatorname{Pt}(111)$ surface (specifically on a Pt(997) surface) the easy axis was found to be perpendicular to the wire direction and under an angle of $43^{\circ}$ with the terrace normal pointing in the direction of the upper terrace [1].

In a later series of experiments, two- and morestranded Co chains were grown along these $\mathrm{Pt}$ step-edges. Measuring the magnetization along different directions, Gambardella et al.[2] found a reorientation of the easy axis from $46^{\circ}$ for the single-stranded wire to $-60^{\circ}$ for the doublestranded one and $-61^{\circ}$ for the four-stranded wire, to finally almost perpendicular to the vicinal surface for more than one monolayer coverage. These oscillations of the easy axes were accompanied by a quite unexpected variation of the MAE. For a double-wire, the MAE at $45 \mathrm{~K}$ dropped from 2.0 
to merely $0.33 \mathrm{meV} / \mathrm{Co}$ atom, but then went up again to $0.45 \mathrm{meV} / \mathrm{Co}$ atom for the triple-wire and finally, for more than a full monolayer coverage, the MAE decreases again to $0.15 \mathrm{meV} / \mathrm{Co}$ atom at $262 \mathrm{~K}$. (Depending on the method how to extract these numbers from the experimental magnetization curves, this variation can be even larger.) So, instead of a continuous decrease, as one could expect from the analysis of Co clusters of different size on $\mathrm{Pt}(111)$ [3], one observes an oscillation. Tight-binding calculations of unsupported chains of different width have already indicated the possibility of such oscillatory behavior [4].

The aim of this communication is now to identify the underlying mechanism that causes these variations in MAE and the directions of the easy axis. We present first-principles calculations that show that the observed behavior is already present without taking into account possible relaxations or dislocations, that could also give rise to the nonmonotonic behavior of the MAE and easy axes. Instead, we find a competition of influences from the row at the step-edge and rows that are adjacent to the Pt part of the terrace. Using a simple model to motivate the different behavior of these rows, we can finally simulate the complex behavior of the magnetic properties in these systems.

\section{Structural model and computational method}

As a model for the stepped surfaces, we calculated vicinal $\mathrm{Pt}(111)$ surfaces of a terrace width of 6 atomic rows. On a (111) surface, a $\langle 111\rangle$ microfaceted (or B-type) with a terrace width of $p$ full atomic rows is notated by $(p, p, p-2)$. Therefore, we calculated films of $\mathrm{Pt}(664)$ surfaces with a thickness of about 8 layers of fcc (111)-type, resulting in total in 45 atoms per unit cell. For the simulation of the wires, we substituted the Pt atoms starting from the edge by Co.

The calculations are based on the (spin) density functional theory in the generalized gradient approximation [5]. We used the full-potential linearized augmented plane wave (FLAPW) [6] method in the FLEUR implementation. Spin-
Table 1

(Spin) Magnetic moments (in $\mu_{\mathrm{B}}$ ) of an unsupported Co chain, supported $n$-chains $(n=1-6)$ on $\mathrm{Pt}(664)$ and $\mathrm{Co}$ monolayers on $\operatorname{Pt}(111)$. In the columns $1-6$, the topmost number always corresponds to the step-edge atom.

\begin{tabular}{ccccccccc}
\hline unsupp. & \multicolumn{6}{c}{ Co/Pt(664) } & \multicolumn{4}{c}{ Co/Pt(111) } \\
chain & 1 & 2 & 3 & 4 & 5 & 6 & fcc & hcp \\
\hline 2.33 & 2.14 & 2.08 & 2.08 & 2.08 & 2.08 & 2.04 & 2.06 & 2.07 \\
& & 2.09 & 2.05 & 2.05 & 2.04 & 2.02 & & \\
& & & 2.09 & 2.04 & 2.04 & 2.04 & & \\
& & & 2.09 & 2.05 & 2.04 & & \\
& & & 2.09 & 2.06 & & \\
& & & & \\
& & &
\end{tabular}

orbit coupling was included as described in Ref.[8]. For all structures the calculated $\mathrm{Pt}(111)$ in-plane lattice constant $(2.82 \AA$, the experimental value is $2.77 \AA$ ) was used. For these calculations approx. 80 basisfunctions/atom and $5 \mathbf{k}_{\|}$-points in the irreducible part of the surface Brillouin zone (2D-BZ) have been used. For the calculation of the magnetocrystalline anisotropy energy (MCA) with the magnetic force-theorem we started from an self-consistent calculation with spin-orbit coupling included and then used $128 \mathbf{k}_{\|}$-points in the full 2D-BZ. The applicability of this procedure was checked in Ref. [9]. No relaxations were included in the present calculations. An investigation of their influence on the easy axis for a single Co chain on $\mathrm{Pt}(664)$ can also be found in Ref. [9].

The magnetic anisotropy energy (MAE) is a sum of the MCA and the shape anisotropy (SA). Taking only the latter into account, in a single stranded wire the hard axis would be in a plane perpendicular to the wire direction. In flat, multi-stranded wires the SA puts the medium axis in the plane of the stripe formed by the strands of the wire, i.e. it causes an additional anisotropy in the plane perpendicular to the wire. In our case this anisotropy is in the order of $0.1 \mathrm{meV} /$ atom, i.e. in most cases much smaller than the MCA. Therefore, the SA will not be considered in the following.

\section{Results}

When we compare the spin magnetic moments, $\mu_{s}$, of the chains of different width (table 1 ), we 


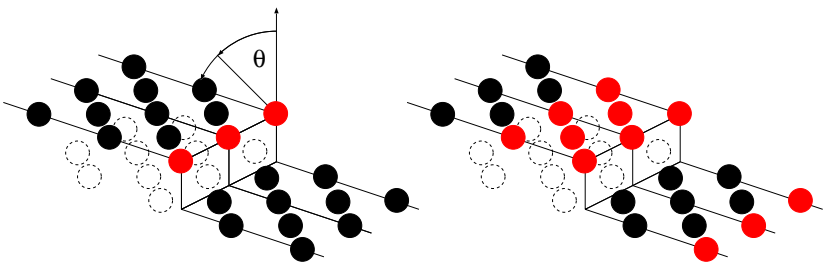

Fig. 1. Schematic drawing of a (111) B-type step-edge decorated with one (left) and three chains (right). In the calculations the spin-quantization axis was varied in a plane perpendicular to the wire by an angle $\theta$ as indicated on the left.

notice that the single Co wire at the Pt step-edge has a magnetic moment that is larger than the moment of a Co monolayer on Pt(111), but substantially smaller than the unsupported monowire. Chains that consist of more than one strand have magnetic moments that are very similar to that of the supported monolayer. For $n$-chains with $n=$ $2-5$, we see that the step-edge atom always has a slightly higher moment of $2.08 \mu_{\mathrm{B}}$, as could be expected from its lower coordination number. Also the innermost strand, that has more Pt nearestneighbors than the other strands, has a higher magnetic moment of $2.09 \mu_{\mathrm{B}}$, while the inner Co-rows show moments of 2.04 or $2.05 \mu_{\mathrm{B}}$. The spin magnetic moments 6 -chain differs from these findings, which is understandable, since on a (664) vicinal surface the 6 -chain corresponds to a complete Co overlayer. Although the variations of the spin moments of the $n$-chains for $n>1$ are very small, the trends concluded from these values should be reflected quite reliably.

Now we turn to the analysis of the magnetocrystalline anisotropy energy (MAE) of the chains of different width. In Fig. 2 we show the variation of the energy as a function of magnetization direction or, more precise, the spin-quantization axis imposed by the calculation. The variation of the magnetization direction was restricted to a plane perpendicular to the wire directions (these were also the experimentally investigated directions). We characterize the direction by an angle $\theta$ which is zero for the surface normal and positive for directions pointing towards the upper terrace (Fig. 1). For the deposited Co monowire, we find the already reported results [9] of a rather large MAE of almost $2.5 \mathrm{meV}$ and an easy axis that is tilted

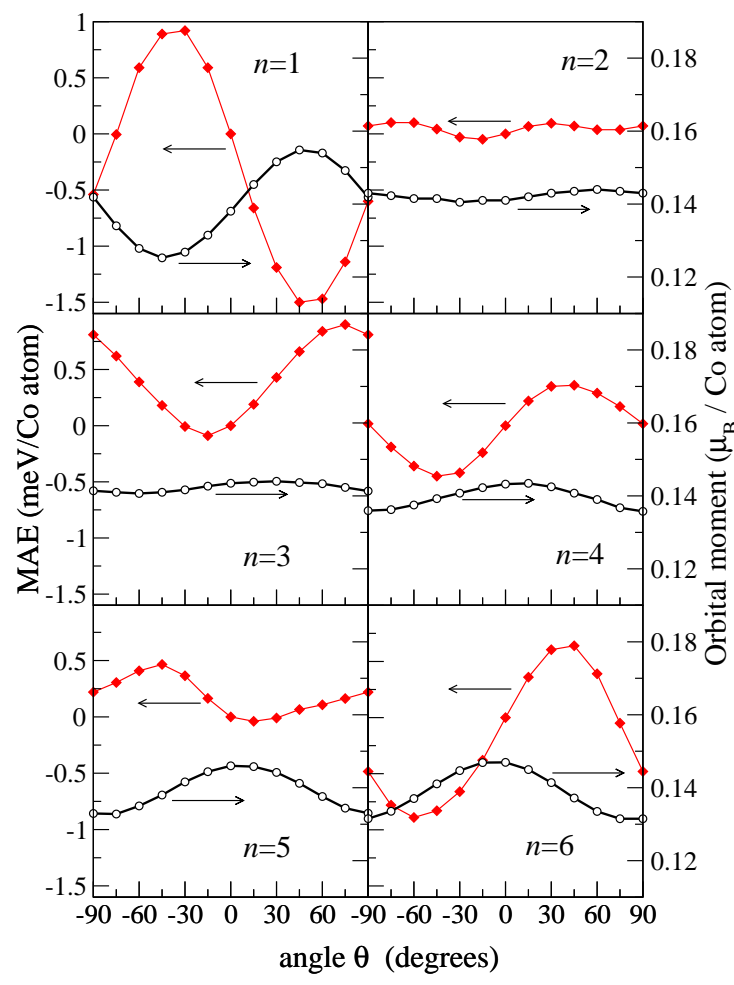

Fig. 2. (color online) Magnetocrystalline anisotropy energy (MAE, full diamonds) and average orbital moment on $\mathrm{Co}$ (empty circles) for Co chains of different width (denoted by $n$ ) on $\mathrm{Pt}(664)$. Note the different scales for MAE (left) and orbital moments (right).

by $51^{\circ}$ with respect to the surface normal in the direction of the upper terrace. In sharp contrast to this result, the Co double-wire shows a much smaller magnetic anisotropy $(0.15 \mathrm{meV})$ and an easy axis with an angle $\theta=-19^{\circ}$, i.e. pointing away from the upper terrace. This drop of MAE is also accompanied by a decrease of orbital moment anisotropy, i.e. the average orbital moment of the double chain is almost constant as a function of the angle $\theta$. The $n$-chains with $n=3-5$ show again larger magnetic anisotropies and the direction of the easy axis varies from $-15^{\circ}$ for the 3 -wire to $-40^{\circ}$ and $+14^{\circ}$ for the 4 - and 5 -wire. As we increase the numbers of strands beyond 3 , the MAE decreases again, from $0.99 \mathrm{meV}$ for the 3 -wire, to $0.82 \mathrm{meV}$ and $0.50 \mathrm{meV} / \mathrm{Co}$ atom for the 4 - and 5 wires, respectively. The 6 -wire is again rather UNtypical in this sequence and has a high anisotropy $(1.55 \mathrm{meV})$ and an easy axis of $-58^{\circ}$. 
When we compare these results to the experimental data, we find an astonishing agreement in the trends: the pronounced drop MAE and in orbital moment when going from the monowire to the double-wire, then again the unexpected increase of the MAE for the 4-wire, finally a decrease of these values as the coverage increases further. Experimentally the MAE drops from about $2 \mathrm{meV} / \mathrm{Co}$ atom for the monowire to $0.33 \mathrm{meV}$ for the doublewire and increases to $0.45 \mathrm{meV}$ for the 4 -wire, the calculated results are $2.5 \mathrm{meV}, 0.15 \mathrm{meV}$ and $0.82 \mathrm{meV}$ for the 1-, 2- and 4-wires, respectively. Also the oscillation in the direction of the easy axis from positive $\left(+51^{\circ}\right.$ in the calculation and $43^{\circ}$ in the experiment) to negative angles for the 2- and 4wires $\left(-19^{\circ}\right.$ and $-40^{\circ}$ as compared to about $-60^{\circ}$ experimentally) is reproduced. Although there is no perfect quantitative agreement (which cannot be expected e.g. due to the different terrace-width), we nevertheless assume that the calculation captures the essential physical mechanism that lies behind the oscillations observed experimentally. Therefore, we will now use our calculations to elucidate this mechanisms at least tentatively.

Employing our $a b$ initio calculations we can analyze the orbital moments of the different strands of the $n$-wires as a function of the spin-quantization axis individually. Looking at the orbital moments of the different strands in the $n$-chains (Fig. 3 ) we find for $n=2-5$ again similarities like we found for the spin moments (table 1 ). The step-edge atom and the innermost strand of the wire behave very similar in all these $n$-wires: The orbital moment at the step-edge atom is the largest of all Co atoms (due to the lower coordination as an atom at the step-edge) and reaches its maximum at small positive angles $\theta$ of the direction of the spin quantization axis. At this angle, the atoms of the innermost strand of the wires have the smallest orbital moment as a function of the angle $\theta$, and their average moment is smaller (around $0.12 \mu_{B}$ as compared to about $0.16 \mu_{B}$ for the step-edge atom). The average orbital moment of the other strands (for the 3to 6 -wires) are similar to the one of the innermost strand, since the coordination number is also identical, but - in contrast - it reaches its maximum at a similar $\theta$-value as the step-edge atom.

When we characterize the individual strands $i$

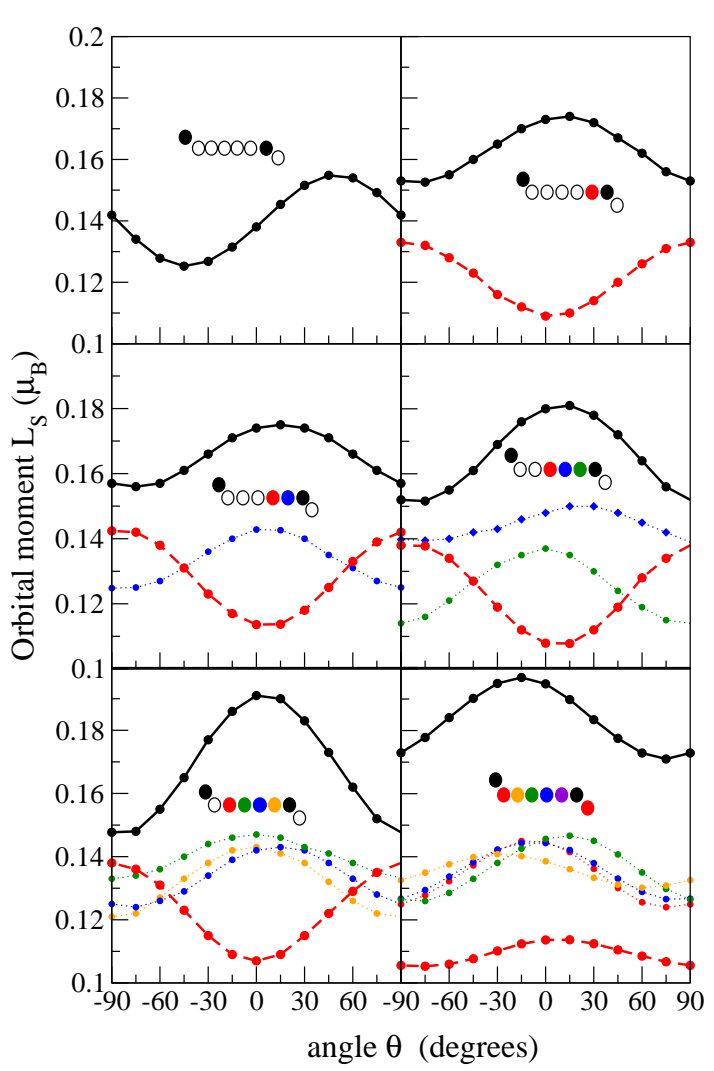

Fig. 3. Orbital moments of the different strands of Co $n$-chains on $\mathrm{Pt}(664)$. The orbital moment of the strand at the step-edge is indicated by the thick full line, the orbital moments of the innermost strand are connected by a thick broken line. Moments of the strands in-between are connected by thin dotted lines. The insets show the surface of the Pt substrate with $\mathrm{Pt}$ atoms as empty circles and Co chains in color.

of an $n$-wire by their nearest neighbors, we identify four groups: the step-edge atom $(i=1)$ which has seven nearest neighbors of which two $(n=$ $1)$ or four $(n>1)$ are Co atoms, the atoms of the innermost strand $(i=n>1)$ which have nine nearest neighbors of which four are Co, and the other strands $(n>i>1)$, where the atoms also have nine nearest neighbors, but now six of them are Co (cf. Fig. 1). From Fig. 3 we see, that each of these four groups shows a characteristic behavior of the orbital moment as a function of $\theta$. Let us look at the potential that acts on the electrons in an atom of a given strand: in all cases they will experience a potential from two neigh- 
boring Co atoms of the same strand, and from the lower-lying three Pt nearest neighbors. Left and right of the strand, we can find either two Pt, or two Co atoms or vacuum. So we characterize our four groups according to the neighboring strands as (Pt:Vac), (Co:Vac), (Pt:Co) and (Co:Co). Assuming that the Pt-potential is strong it will - in the spirit of Ref. [10] - favor an in-plane magnetization (or at least a large in-plane orbital moment at the neighboring Co atoms). The smaller Co atoms, which are spaced by the large Pt lattice constant, produce a weaker potential that favors a large out-of-plane orbital moment (like it is observed in a Co monolayer on $\operatorname{Pt}(111)$ ). Thus, we can find a natural explanation of the variation of the orbital moment observed in Fig. 3.

We can now employ a simple model to relate these variations in orbital moments to the magnetocrystalline anisotropy energy. Suppose, that the MAE $(\Delta E)$ can be written as a sum of contributions of the different strands $i$ in the wire: $\Delta E=$ $\sum_{i}^{n} \xi_{i} \mathbf{S}_{i} \cdot \Delta \mathbf{L}_{i}$. Here, $\xi_{i}$ is the spin-orbit coupling constant of the atoms of strand $i$ (which we can assume to be constant for all $n$ and $i), \mathbf{S}_{i}$ are the spin moments (cf. Table 1) and $\Delta \mathbf{L}_{i}$ is the orbital moment anisotropy (OMA). For a monowire, the OMA and the MAE correspond nicely: where the orbital moment is highest, the energy has its minimum. For a double-wire, the contributions of the two strands almost cancel each other, so that the average OMA is very small (cf. Fig. 2), therefore also the MAE is extremely small. Indeed, we find that the MAE curve has two shallow minima and it seems that it is the contribution of the step-edge atom that finally makes the minimum at a small negative angle slightly deeper than the minimum at larger positive ones. (In this case the shape anisotropy can not be neglected and favors even larger angles). When the number of strands is larger than two, the innermost strand and the step-edge strand still compensate each other, but the contribution of the remaining strands gives now rise to a larger MAE and favors an easy axis at negative values of $\theta$. Interestingly, in the case of a 5-chain, the easy axis turns once more towards positive angles. At larger chain width it is difficult to compare with experimental data, since our terraces are $33 \%$ narrower than in the experiment.

\section{Summary}

We presented $a b$ initio calculations of Co chains of different width deposited on a stepped Pt surface. In agreement with experimental observations the easy axis oscillates as a function of chain thickness around the terrace normal and the MAE shows a non-monotonic behavior. We attribute these findings to the individual contributions of the Co chains to the MAE and the total orbital moment. Depending on the position of the Co chain (at the step-edge or in the terrace), these contributions can be very different and cancel each other, resulting in a complex behavior of the magnetic properties.

\section{ACKNOWLEDGMENTS}

G.B. gratefully acknowledges Marcus Heide for supplying a code to calculate the shape anisotropies of $n$-chains.

\section{References}

[1] P. Gambardella, A. Dallmeyer, K. Maiti, M. C. Malagoli, W. Eberhardt, K. Kern, and C. Carbone, Nature 416, 301 (2002).

[2] P. Gambardella, A. Dallmayer, K. Maiti, M. C. Malagoli, S. Rusponi, P. Ohresser, W. Eberhardt, C. Carbone, and K. Kern, Phys. Rev. Lett. 93, 077203 (2004).

[3] P. Gambardella, S. Rusponi, M. Veronese, S. S. Dhesi, C. Grazioli, A. Dallmeyer, I. Cabria, R. Zeller, P. H. Dederichs, K. Kern, et al., Science 300, 1130 (2003).

[4] J. Dorantes-Dávila and G. M. Pastor, Phys. Rev. Lett. 81, 208 (1998).

[5] J. P. Perdew, J. A. Chevary, S. H. Vosko, K. A. Jackson, M. R. Pederson, D. J. Singh, and C. Fiolhais, Phys. Rev. B 46, 6671 (1992).

[6] E. Wimmer, H. Krakauer, M. Weinert, and A. Freeman, Phys. Rev. B 24, 864 (1981).

[7] For program description see http://www.flapw.de.

[8] C. Li, A. J. Freeman, H. J. F. Jansen, and C. L. Fu, Phys. Rev. B 49, 5433 (1990).

[9] S. Baud, C. Ramseyer, G. Bihlmayer, and S. Blügel, Phys. Rev. B p. submitted (2005).

[10] J. Stöhr, J. Magn. Magn. Mater. 200, 470 (1999). 